\title{
Effects of selective serotonin reuptake inhibitors on rating-scale-assessed suicidality in adults with depression
}

\author{
Jakob Näslund, Fredrik Hieronymus, Alexander Lisinski, Staffan Nilsson and Elias Eriksson
}

\section{Background}

Selective serotonin reuptake inhibitors (SSRIS) have been claimed to elicit or aggravate suicidal ideation.

\section{Aims \\ To explore the effect of SSRIS on the suicidality item of the Hamilton Rating Scale for Depression (HRSD).}

\section{Method}

We undertook a patient-level mega-analysis of adults with depression participating in industry-sponsored studies of sertraline, paroxetine or citalopram, comparing patients on an SSRI $(n=5681)$ with those on placebo $(n=2581)$ with respect to HRSDrated suicidality. Separate analyses were conducted for young adults (age $18-24 ; n=537$ ) and adults (age $\geq 25 ; n=7725$ )

\section{Results}

Among adults, the reduction in mean rating of suicidality was larger and the risk for aggravation of suicidality lower in patients receiving an SSRI from week 1 and onwards. In young adults, SSRI treatment neither reduced nor increased suicidality ratings relative to placebo at the end-point.

\section{Conclusions}

The net effect of SSRIs on suicidality appears beneficial in people above the age of 24 and neutral in those aged 18-24.

\section{Declaration of interest}

F.H. has received speaker's fees from Servier. E.E. has previously been on the advisory boards and/or received speaker's honoraria and/or research grants from Eli Lilly, GlaxoSmithKline, Servier and Lundbeck.

\section{Copyright and usage}

(c) The Royal College of Psychiatrists 2018.
The possibility that selective serotonin reuptake inhibitors (SSRIs) may elicit or aggravate suicidal ideation ${ }^{1}$ remains controversial, some claiming such an effect to be relatively common, ${ }^{2,3}$ others finding merely weak ${ }^{4}$ or no ${ }^{5-11}$ support for it, and yet others suggesting the net effect of SSRIs on suicidality to be beneficial. ${ }^{12-17}$ One strategy to shed light on this issue is to investigate the frequency of actual suicides or suicide attempts in patients receiving SSRIs or placebo, respectively, in controlled trials. Interpretation of this kind of data is, however, rendered difficult because of the rarity of relevant events and uncertainty with respect to how possibly relevant events have been classified, ${ }^{18}$ moreover, the literature on this issue is far from unanimous, some reporting suicide and suicide attempts to be more common in individuals taking SSRIs ${ }^{3}$ and others not finding such a difference. ${ }^{8,9,11}$

Another strategy to assess the possible suicide-provoking effect displayed by SSRIs in placebo-controlled trials, which could be regarded as complementary to the investigation of actual suicides or suicide-related adverse events, is to analyse the effect of treatment on rating-scale-assessed suicidal ideation in individual patients. ${ }^{7,12,13}$ Using this strategy, one may both obtain a measure of the net influence of treatment on suicidality at a group level as well as detect individual cases of emergence or aggravation of suicidal ideation. Whereas analyses of rating-scale-assessed suicidality have been published regarding fluoxetine ${ }^{12,13}$ and venlafaxine ${ }^{13}$ there has, to the best of our knowledge, not been any comprehensive analyses regarding the effect of other serotonergic antidepressants on suicidality using this approach. We therefore decided to analyse patient-level data from all Food and Drug Administration (FDA)-registered, industry-sponsored, Hamilton Rating Scale for Depression (HRSD)-based, placebo-controlled trials that have been conducted to evaluate the effects of citalopram, paroxetine and sertraline in adults with depression, with respect to the effect of treatment on suicidal ideation as assessed using item 3 of the
HRSD. ${ }^{7}$ Prompted by the suggested importance of age for a possible suicide-provoking effect of SSRIs, children, adolescents and young adults tentatively being more prone to experience SSRI-elicited suicidal ideation, ${ }^{13,19-22}$ all analyses were carried out separately in patients aged 18-24 (young adults) and those aged 25 and above (adults).

\section{Method}

\section{Data acquisition and participants}

We requested and obtained patient-level symptom rating data for all industry-sponsored, HRSD-based, FDA-registered placebo-controlled studies undertaken to explore the effects of citalopram, paroxetine or sertraline in major depression in adults. By inspecting the FDA approval packages ${ }^{23-26}$ we could confirm that we had access to all pertinent studies with the exception of three small trials that had been prematurely terminated: GSK/07 $(n=25), \mathrm{LB} / 86 \mathrm{~A}(n=24)$ and LB/87A $(n=34)$. In addition, GSK and Pfizer provided patient-level data from ten post-registration or post-marketing trials that were also included in the analyses. In three studies (GSK/115, GSK/128 and PZ/111), an SSRI, fluoxetine, had been used as the active comparator; patients receiving this drug were also included in the analyses whereas patients given a non-SSRI comparator were not. The trials included in the analyses are presented in supplementary Table 1 (available at https://doi.org/10. 1192/bjp.2017.24).

All analyses were undertaken using data that had been pooled, SSRI (regardless of drug and dose) and placebo constituting the groups to be compared. Except for when otherwise stated, young adults $(18-24)(n=537)$ and adults $(\geq 25)(n=7725)$ were analysed separately. The Regional Ethical Review Board of Gothenburg, 
Sweden issued an advisory opinion stating no objection to the conduct of this study.

\section{Analyses of effects of treatment on mean item 3 rating}

The effect of treatment on the mean rating of suicidal ideation (HRSD item 3) was analysed using a linear mixed model with study, treatment, time (week) and the time $\times$ treatment interaction as categorical predictors and baseline rating as a covariate. The correlated observations were modelled as a repeated effect using an unstructured covariance matrix. All post-baseline weekly ratings up to and including week 6 were included in the model. Two sensitivity analyses were undertaken: (a) a last-observation-carriedforward ANCOVA with treatment and study as fixed factors and baseline rating as a covariate (intention-to-treat (ITT) group) and (b) an analysis only comprising patients discontinuing before the end-point assessment using the same ANCOVA model (drop-out group). Additionally, we compared the baseline ratings of suicidal ideation between age groups, regardless of treatment (supplementary Fig. 1(a)), and also between treatments within age groups at baseline using $t$-tests.

Prompted by apparent differences in response patterns between the two age groups, we performed two additional follow-up analyses in order to compare participants aged 18-24 with those $\geq 25$ years of age with respect to suicidal ideation at the end-point and at the last available observation (supplementary Fig. 1(b) and (c)). For these analyses an ANCOVA model with age group, treatment and the interaction between age group and treatment as fixed factors, and the baseline rating of suicidal ideation as a covariate, was used.

\section{Analyses of effects of treatment on the prevalence of item 3 aggravation}

To explore the risk of experiencing an increase in suicidal ideation, three categorical definitions of deterioration were explored: (a) a higher rating of item 3 during treatment than at baseline ('worsening'), (b) an item 3 rating of 2-4 during treatment in a participant with a rating of 0 or 1 at baseline ('emergent suicidality: loose definition') $)^{27,28}$ and (c) an item 3 rating of 3-4 during treatment in a participant with rating of 0 or 1 at baseline ('emergent suicidality: strict definition'). ${ }^{29,30}$ Patients that because of their baseline score were unable to experience deterioration according to each definition were excluded from the corresponding analyses. For each of the three definitions of deterioration, differences between treatment groups in participants aged $\geq 25$ and $18-24$, respectively, were assessed using Kaplan-Meier procedures.

Since some reports suggest the risk for an SSRI-induced increase in suicidal ideation to be at its highest shortly after the onset of treatment, separate analyses were conducted for the early phase of treatment (weeks 1-2) and for the remainder of the treatment period (weeks 3-6), respectively using logistic regression. Patients that, because of their baseline score, were unable to experience deterioration according to each definition were excluded from the corresponding analyses; participants who dropped out who were unable to show deterioration because of lacking an HRSD evaluation were similarly excluded. Moreover, we assessed the risk of deterioration in patients dropping out before the end-point visit. The model included treatment and study as fixed factors and the baseline rating as a covariate; however, we also provide the unadjusted odds ratios resulting from only including the treatment factor.

Finally, we wished to investigate whether emergent suicidality in patients treated with SSRIs may be dissociated from other symptoms of depression to a larger extent than in those given placebos. SSRItreated patients with emergent suicidality (both definitions) were compared to placebo-treated patients with emergent suicidality with respect to two different assessments at the visit when emergent suicide was first recorded (regardless of when this visit occurred): (a) the rating of the depressed mood item on the HRSD scale and (b) the sum rating of all HRSD symptoms. These analyses were undertaken using $t$-tests (supplementary Tables 2 and 3 ).

\section{Software used}

SAS for Windows, version 9.4 was used to analyse treatment effects on item worsening and item mean scores. SPSS for Mac, version 21 was used for all other statistical procedures.

\section{Results}

\section{Effects of treatment on mean item 3 rating}

In the adult population, the mean rating of suicidal ideation was significantly lower in the patients taking SSRIs than in those given placebos from week 1 and onwards (Fig. 1(a)). In young adults, the mean rating of suicidal ideation was significantly lower in those receiving an SSRI at week 1 but not at subsequent visits (Fig. 1(b)). These findings were mirrored by the results in the two sensitivity populations. Thus, with respect to adults, both the intention-to-treat and the drop-out groups showed significant improvements for patients taking SSRIs of roughly the same magnitude as that obtained in the end-point analysis (Fig. 1(a)). For young adults, there were no significant differences between groups in either of the sensitivity populations (Fig. 1(b)).

The comparison of young adults with adults revealed young adults to score slightly but significantly higher on suicidal ideation at baseline (effect size $(\mathrm{ES})=-0.15, P=0.001$ ) (supplementary Fig. 1(a)). The analysis addressing the effect of age and treatment on end-point rating of suicidal ideation revealed a significant $(P=$ $0.009)$ interaction; whereas there was little difference in rating between young adults and adults taking SSRIs, placebo-treated young adults displayed significantly lower ratings than placebotreated adults (supplementary Fig. 1(b)). The analysis based on the last available observation found similar results (interaction $P=0.04$ ) (supplementary Fig. 1(c)).

\section{Effects of treatment on the prevalence of item 3 aggravation}

The results of the Kaplan-Meier survival analyses are summarised in Fig. 2. In patients aged 25 and above, the risk for suicidal worsening and suicidal emergency (loose definition) was lower in those on an SSRI than in those on placebo; in contrast, there were no significant differences with respect to the strict definition of emergent suicidality. In participants below the age of 25 , there were no significant differences between treatment groups.

The logistic regression regarding participants $\geq 25$ years of age revealed those treated with an SSRI to be less likely to report worsening of suicidal ideation or emergent suicidality (loose definition) than those given placebo when considering weeks 1-2 and weeks 3-6 separately, as well as when including only those patients who did not complete their respective trials (Table 1); again there were, however, no significant differences between groups when emergent suicidality was more strictly defined.

In young adults, those given an SSRI were at enhanced risk for worsening of suicidal ideation (in the unadjusted analysis) or emergent suicidality (loose but not strict definition) during the late (weeks 3-6) but not the early phase (weeks 1-2) of treatment. There were no significant differences between treatments in young adult who did not complete treatment (Table 2).

Neither in young adults nor in adults did we obtain support for the possibility that emergent suicidality would be dissociated from depression severity to a greater extent in the patients taking SSRIs than in those taking placebo (supplementary Table 2 and 3). 

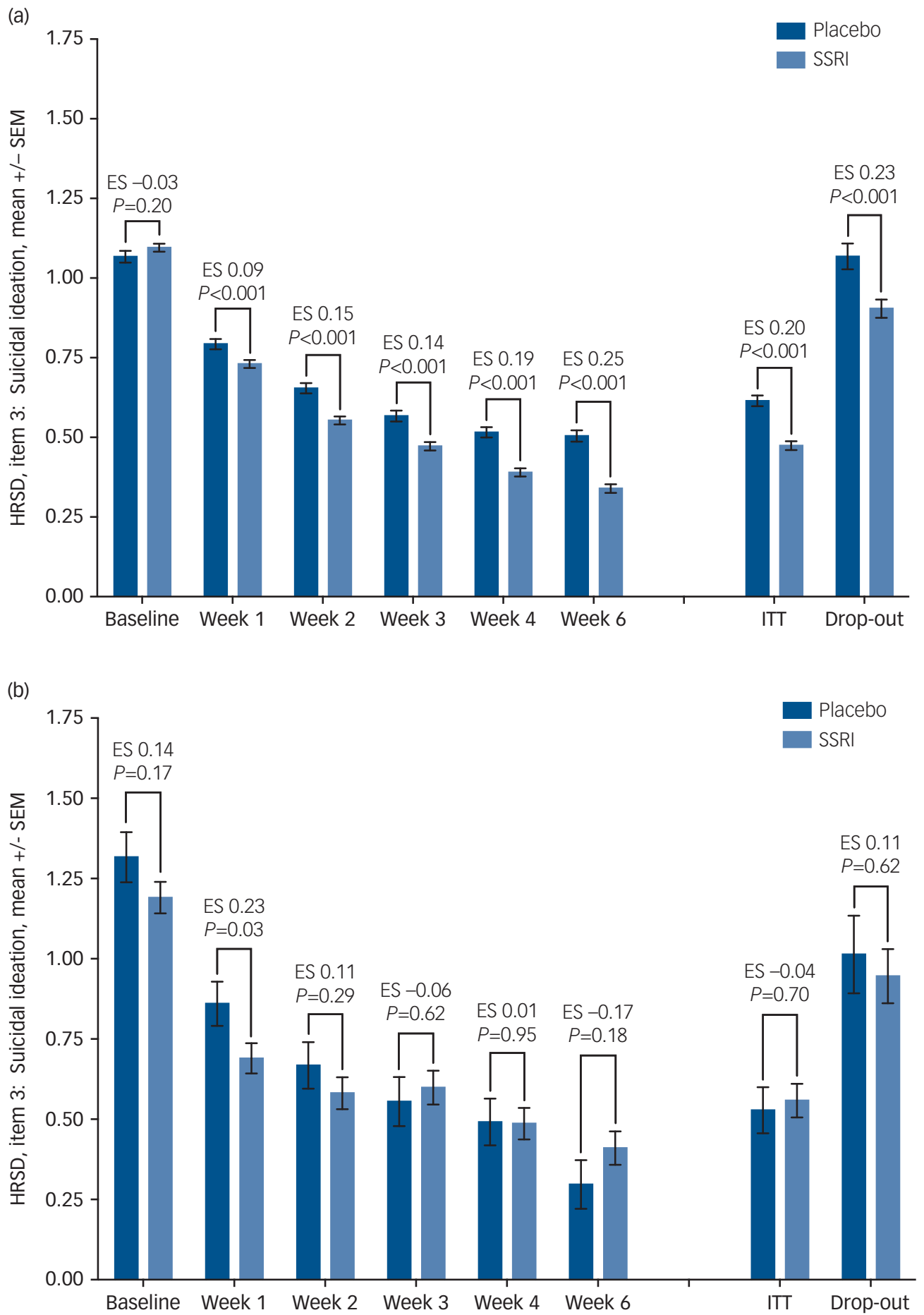

Fig. 1 Rating of suicidality over 6 weeks for patients aged (a) $\geq 25$ and (b) 18-24.

Also shown are the intention-to-treat (ITT) group (last observation carried forward) and the rating of the last visit available for patients dropping out (drop-out group). HRSD, Hamilton Rating Scale for Depression; ES, effect size; SEM, standard error of the mean; SSRI, selective serotonin reuptake inhibitor.

\section{Discussion}

\section{Main findings}

In patients above the age of 24 , SSRIs were found to reduce the mean rating of the HRSD suicidality item from week 1 until study endpoint and also to reduce the risk for aggravation of suicidal ideation and emergent suicidal behaviour. Although it, nevertheless, cannot be discounted that SSRIs in rare cases may exert a suicide-provoking effect in this age group, this study provides no support for this assumption, but strong support for the view that the net effect of SSRI treatment is beneficial rather than harmful. These results are congruent with previous reports showing two other drugs exerting a marked influence on the serotonin transporter (i.e. another SSRI, fluoxetine $\mathrm{e}^{7,12,13}$ and a serotonin and noradrenaline reuptake inhibitor, venlafaxine ${ }^{13}$ ) to reduce rather than enhance HRSD-rated suicidal ideation in placebo-controlled trials. Likewise, they are congruent with register-based epidemiological reports suggesting 
(a) Worsening of suicidal ideation $\geq 25$ years

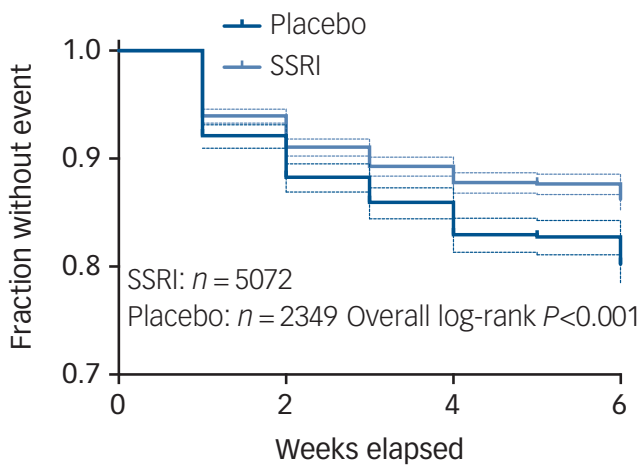

Emergent

(c) suicidality (loose definition) $\geq 25$ years

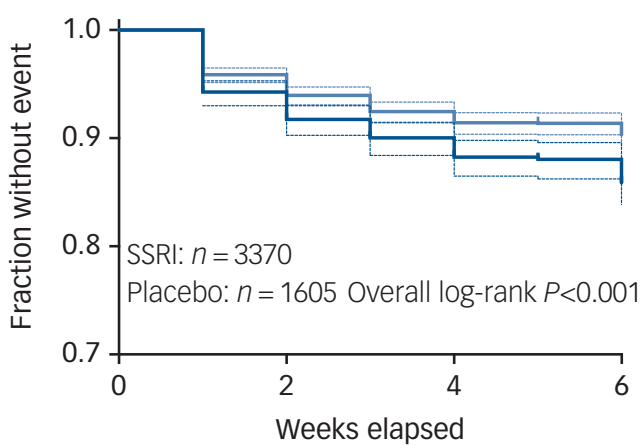

Emergent

(e) suicidality (strict definition) $\geq 25$ years

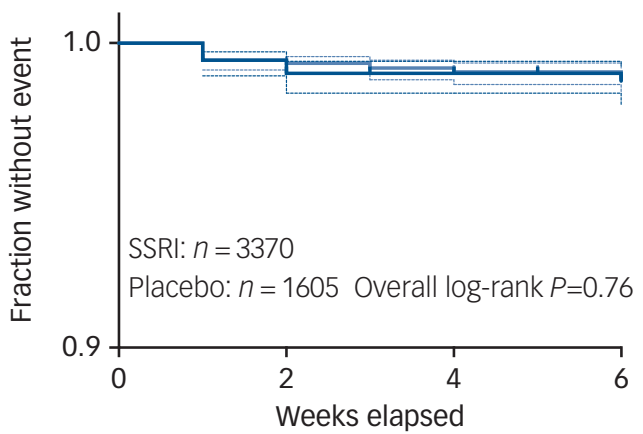

(b) Worsening of suicidal ideation 18-24 years

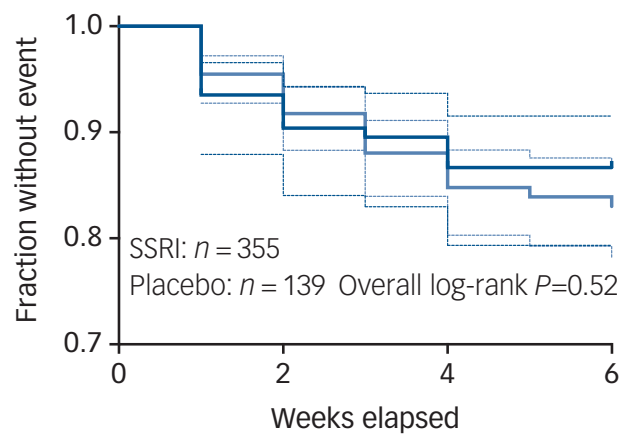

Emergent

(d) suicidality (loose definition) 18-24 years

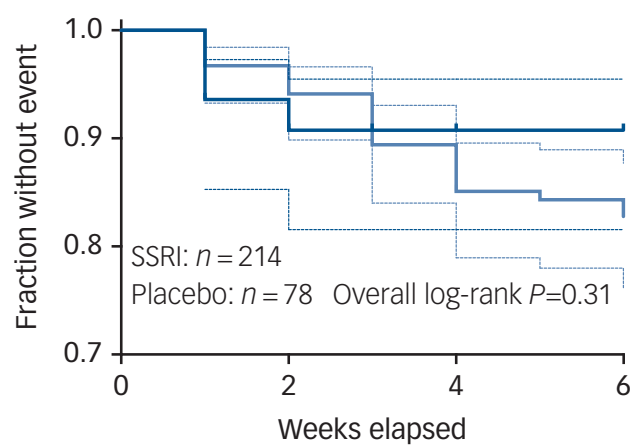

Emergent

(f) suicidality (strict definition) 18-24 years

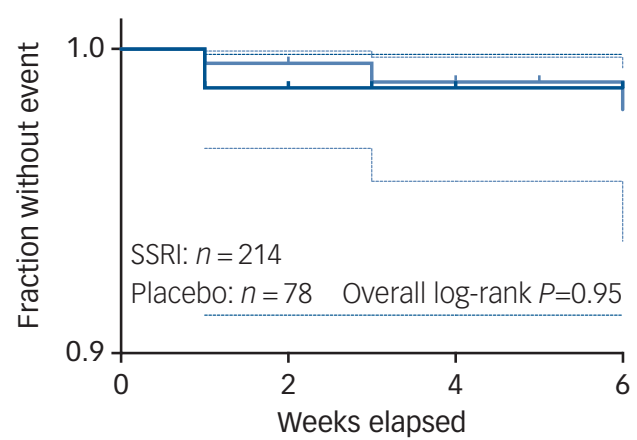

Fig. 2 Instances of enhanced suicidality as assessed using item 3 of the Hamilton Rating Scale for Depression for patients aged $\geq 25$ (a, c and e) and $18-24(b, d$ and $f)$.

Plots indicate event-free survival for each treatment group, dotted lines indicate $95 \%$ confidence intervals. Shown in each graph are the number of patients and the results of overall log-rank tests. (a) Worsening of suicidal ideation $\geq 25$ years of age; (b) worsening of suicidal ideation 18-24 years; (c) emergent suicidality (loose definition) $\geq 25$ years of age; (d) emergent suicidality (loose definition) 18-24 years; (e) emergent suicidality (strict definition) $\geq 25$ years of age; (f) emergent suicidality (strict definition) $18-24$ years. SSRI, selective serotonin reuptake inhibitor

that SSRIs prevent suicide attempts and with analyses of temporal associations between changes in SSRI prescription and suicide frequencies. ${ }^{14,16,17,31}$

\section{Age differences in our material}

Our decision to split the population into those aged 18-24 years on the one hand, and those $\geq 25$ years of age on the other, was prompted by the frequent claim that a suicide-provoking effect of the SSRIs may be more common in children, adolescents and young adults ${ }^{13,19,22}$ than in older individuals; in line with this notion, the FDA and the European Medicines Agency have released controversial $^{32}$ black box warnings regarding prescribing these drugs to individuals below 25 years of age. Although the effect of SSRIs on suicidal ideation in children is beyond the scope of the present paper, in all included trials dealing with depression in adults it is notable that we observed neither a beneficial nor a harmful effect of SSRIs on HRSD-assessed suicidal ideation at the end-point in participants between 18 and 24 years of age. Likewise, when considering the entire study period, there were no significant differences between treatments with respect to KaplanMeier survival curves. However, when individuals with an increase in item 3 rating were analysed using logistic regression, both worsening of suicidal ideation and suicidal emergency (when defined using the less stringent criteria, but not when more strictly 


\begin{tabular}{|c|c|c|c|c|}
\hline & Placebo, \% (n/N) & SSRI, \% (n/N) & Unadjusted OR $(95 \% \mathrm{Cl}) ; P$ & Adjusted $\mathrm{OR}^{\mathrm{b}}(95 \% \mathrm{Cl}) ; P$ \\
\hline \multicolumn{5}{|l|}{ Worsening of suicidal ideation ${ }^{c}$} \\
\hline Week 1-2 & $11.6(271 / 2346)$ & $8.7(441 / 5066)$ & $0.73(0.62-0.86) ;<0.001$ & $0.66(0.56-0.79)_{i}<\mathbf{0 . 0 0 1}$ \\
\hline Week 3-6 & $12.6(260 / 2060)$ & $7.7(333 / 4300)$ & $0.58(0.49-0.69) ;<0.001$ & $0.52(0.43-0.63) ;<0.001$ \\
\hline Week 1-6 (drop-out group) ${ }^{d}$ & $18.7(129 / 689)$ & $12.6(178 / 1412)$ & $0.63(0.49-0.80) ;<0.001$ & $0.54(0.41-0.72) ;<0.001$ \\
\hline \multicolumn{5}{|c|}{ Emergent suicidal ideation: loose definition ${ }^{\mathrm{e}}$} \\
\hline Week 1-2 & $8.2(131 / 1605)$ & $6.0(203 / 3370)$ & $0.72(0.57-0.91) ; \mathbf{0 . 0 0 5}$ & $0.66(0.51-0.84) ;<0.001$ \\
\hline Week 3-6 & $9.2(131 / 1418)$ & $5.9(171 / 2893)$ & $0.62(0.49-0.78) ;<0.001$ & $0.55(0.42-0.71) ;<0.001$ \\
\hline Week 1-6 (drop-out group) ${ }^{d}$ & $15.5(67 / 433)$ & $9.5(83 / 877)$ & $0.57(0.40-0.81) ; \mathbf{0 . 0 0 1}$ & $0.49(0.33-0.73) ;<\mathbf{0 . 0 0 1}$ \\
\hline \multicolumn{5}{|c|}{ Emergent suicidal ideation: strict definition ${ }^{f}$} \\
\hline Week 1-2 & $1.1(18 / 1605)$ & $0.8(28 / 3370)$ & $0.74(0.41-1.34) ; 0.31$ & 0.57 (0.30-1.08); 0.09 \\
\hline Week 3-6 & $1.3(19 / 1418)$ & $0.9(27 / 2893)$ & $0.69(0.38-1.25) ; 0.22$ & $0.68(0.36-1.27) ; 0.22$ \\
\hline Week 1-6 (drop-out group) ${ }^{d}$ & $2.5(11 / 433)$ & $1.8(16 / 877)$ & $0.71(0.33-1.55) ; 0.39$ & $0.47(0.19-1.12) ; 0.09$ \\
\hline \multicolumn{5}{|c|}{$\begin{array}{l}\text { Results in bold are significant. } \\
\text { SSRI, selective serotonin reuptake inhibitor. } \\
\text { a. Shown are events occurring during the early (week 1-2) and late (week 3-6) phases, respectively, as well as events occurring at any time in the population that discontinued prematurely. } \\
\text { b. Adjusted for trial and baseline HRSD item } 3 \text { rating. } \\
\text { c. Worsening of suicidal ideation defined as a higher HRSD item } 3 \text { rating as compared with baseline. } \\
\text { d. Patients without a week } 6 \text { visit or without a week } 4 \text { visit in studies lacking a scheduled visit at week } 6 \text {. } \\
\text { e. Emergent suicidal ideation: loose definition defined as an HRSD item } 3 \text { rating of } 2-4 \text { in participants with a rating of } 0 \text { or } 1 \text { at baseline. } \\
\text { f. Emergent suicidal ideation: strict definition defined as an HRSD item } 3 \text { rating of } 3 \text { or } 4 \text { in participants with a rating of } 0 \text { or } 1 \text { at baseline. }\end{array}$} \\
\hline
\end{tabular}

defined) were more common in young adult patients taking SSRIs in the later stages of treatment; no corresponding effect was, however, observed during the first 2 weeks or in the drop-out group. Although this possible sign of an SSRI-induced aggravation is noteworthy, the high number of tests conducted, combined with the relatively weak level of significance, necessitates that this finding be interpreted with caution. On the other hand, it should be considered that the number of participants, and hence the statistical power, was considerably lower in the group of young adults.

The baseline rating of suicidal ideation was slightly higher in young adults than in adults $(P=0.001)$ (supplementary Fig. $1(\mathrm{a}))$. Suicidal ideation in young adults, however, declined, irrespective of treatment, to be roughly on par with that of adults treated with SSRIs at the end-point and at the last available observation (supplementary Fig. 1(b) and (c)). The major difference between the two age groups with respect to the effect of treatment hence appears to be that young adults are more inclined than older participants to display reduced suicidality at the end-point when given placebo, rather than less inclined to do so when given an SSRI. Although our results thus provide no strong support for suicidal aggravation being a common phenomenon in young adults, they also do not provide support for an antisuicidal effect of the SSRIs in this age group.

However, with respect to both age groups, it should be taken into consideration that participants with marked suicidality at baseline are usually excluded from participating in placebo-controlled trials; the number of participants with a baseline rating of 3 or 4 on the HRSD suicide item (rated $0-4$ ) was thus $<6 \%$. Although this caveat probably renders it more difficult to detect a suicidality-reducing signal, it also renders this data-set unsuitable for exploring the possibility that SSRIs may enhance proneness for suicide in individuals displaying marked suicidal tendencies before treatment.

\section{Interpretation of our findings}

The alleged suicide-provoking effect of SSRIs has often been described not as a manifestation of aggravated depression but as a distinct and specific phenomenon. ${ }^{1}$ Tentatively, such an effect might be masked by the antidepressant effect of SSRIs making fewer participants in this group display emergent suicidality because of aggravated depression. If this were the case, it could be expected that the participants in the placebo group who reported

Table 2 Worsening of suicidality and emergent suicidal ideation as assessed using item 3 of the Hamilton Rating Scale for Depression (HRSD) in young adults (18-24 years of age) ${ }^{\mathrm{a}}$

\begin{tabular}{|c|c|c|c|c|}
\hline & Placebo, \% $(n / N)$ & SSRI, $\%(n / N)$ & Unadjusted OR (95\% Cl); $P$ & Adjusted $^{\mathrm{b}}$ OR $(95 \% \mathrm{Cl}) ; P$ \\
\hline \multicolumn{5}{|c|}{ Worsening of suicidal ideation ${ }^{c}$} \\
\hline Week 1-2 & $9.4(13 / 139)$ & $7.9(28 / 355)$ & $0.83(0.42-1.65) ; 0.60$ & $0.69(0.32-1.50) ; 0.35$ \\
\hline Week 3-6 & $5.9(7 / 118)$ & $12.8(38 / 296)$ & $2.34(1.01-5.39) ; \mathbf{0 . 0 4}$ & $2.22(0.90-5.49) ; 0.08$ \\
\hline Week 1-6 (dropouts) ${ }^{d}$ & $10.4(5 / 48)$ & $12.9(16 / 124)$ & $1.27(0.44-3.69) ; 0.66$ & $1.18(0.27-5.13) ; 0.83$ \\
\hline \multicolumn{5}{|c|}{ Emergent suicidal ideation: loose definition ${ }^{e}$} \\
\hline Week 1-2 & $9.0(7 / 78)$ & $6.5(14 / 214)$ & $0.71(0.28-1.83) ; 0.48$ & $0.48(0.17-1.38) ; 0.17$ \\
\hline Week 3-6 & $3.1(2 / 65)$ & $14.8(27 / 182)$ & $5.49(1.27-23.8) ; 0.01$ & $7.35(1.57-34.4) ; \mathbf{0 . 0 1}$ \\
\hline Week 1-6 (dropouts) ${ }^{d}$ & $10.3(3 / 29)$ & $15.3(11 / 72)$ & $1.56(0.40-6.07) ; 0.75$ & $1.27(0.19-8.36) ; 0.80$ \\
\hline \multicolumn{5}{|c|}{ Emergent suicidal ideation: strict definition ${ }^{f}$} \\
\hline Week 1-2 & $1.3(1 / 78)$ & $1.4(3 / 214)$ & $1.09(0.11-10.7) ;>0.99$ & $0.46(0.03-7.13) ; 0.58$ \\
\hline Week 3-6 & $1.5(1 / 65)$ & $2.8(5 / 182)$ & $1.81(0.20-15.8) ;>0.99$ & $1.28(0.11-14.4) ; 0.84$ \\
\hline Week 1-6 (dropouts) ${ }^{\mathrm{d}}$ & $3.5(1 / 29)$ & $0.0(0 / 72)$ & N/A & N/A \\
\hline \multicolumn{5}{|c|}{$\begin{array}{l}\text { Results in bold are significant. } \\
\text { SSRI, selective serotonin reuptake inhibitor; N/A, not applicable. } \\
\text { a. Shown are events occurring during the early (week 1-2) and late (week 3-6) phases, respectively, as well as events occurring at any time in the population that discontinued prematurely. } \\
\text { b. Adjusted for trial and baseline HRSD item } 3 \text { rating. } \\
\text { c. Worsening of suicidal ideation defined as a higher HRSD item } 3 \text { rating as compared with baseline. } \\
\text { d. Patients without a week } 6 \text { visit or without a week } 4 \text { visit in studies lacking a scheduled visit at week } 6 \text {. } \\
\text { e. Emergent suicidal ideation: loose definition defined as an HRSD item } 3 \text { rating of } 2-4 \text { in participants with a rating of } 0 \text { or } 1 \text { at baseline. } \\
\text { f. Emergent suicidal ideation: strict definition defined as an HRSD item } 3 \text { rating of } 3 \text { or } 4 \text { in participants with a rating of } 0 \text { or } 1 \text { at baseline. }\end{array}$} \\
\hline
\end{tabular}


emergent suicidality would display higher ratings of other symptoms of depression than those displaying emergent suicidality in patients taking SSRIs, suicidal ideation in the former being more closely related to depression severity. We, however, obtained no support for the possibility that suicidal emergency in people taking SSRIs should be different from suicidal emergency in those given placebo in terms of depression severity (supplementary Tables 2 and 3 ).

Given that it is often claimed that the beneficial effect of SSRIs requires weeks of treatment, it is of note that a small but significant reduction in suicidal ideation was observed in adults treated with SSRIs after just 1 week of treatment; likewise we have previously reported on a reduction in depressed mood and psychic anxiety at week 1 in reports based largely on the same patient population. ${ }^{33,34}$ The mechanism of action for these effects is hence not likely, as sometimes suggested, to be one that requires weeks of treatment to set in.

It should be underlined that the aim of this study was not to explore the possible influence of SSRIs on suicidal behaviour but merely on suicidal ideation as assessed using item 3 of the HRSD. Whereas some insight into suicidal behaviour might have been achieved by analysing those given a score of 4 for this item, which often (but not necessarily) indicates that an actual suicide attempt has taken place, such an approach was not feasible given the paucity of such events (placebo: $0.1 \%$, SSRI: $0.1 \%$ ).

\section{Limitations}

Four limitations of this study should be mentioned. First, the analyses were based on trials in adults (of whom only a small minority were below 25 years of age) and this allows no conclusions with respect to the much-debated issue of a possible influence on suicidal ideation in children and adolescents. Second, it should be acknowledged that item 3 of the HRSD is likely not an optimal instrument for measuring proneness for suicide; on the other hand, single-item assessments have been found to show predictive validity in high-risk groups $^{35}$ and the suicide item of the HRSD has been shown to correlate reasonably well with established suicide rating scales. ${ }^{36}$ Moreover, the results obtained indicate this measure to be sufficiently relevant to detect a significant difference between the two treatments as early as after 1 week of treatment.

Third, whereas we found no strong support for SSRIs exerting a suicide-provoking effect in either of the two age groups, and strong support for SSRIs causing a net reduction in suicidal ideation in those $\geq 25$ years of age, it obviously cannot be excluded that SSRIs did enhance suicidal ideation in some participants while dampening it in others, the net effect being that of a reduction in suicidal ideation in spite of the fact that some may have reacted negatively to active treatment. Comparing the two treatment groups with respect to depression severity, however, did not provide support for the assumption that this phenomenon should be dissociated from the underlying disorder in the participants taking SSRIs. Fourth, assessing suicide-related adverse event data, which might have provided complementary information of interest, was not part of the design of this study; such information for the trials included has already been scrutinised in previous publications. ${ }^{8,9}$

In summary, these results confirm previous reports suggesting the net effect of administration of SSRIs in individuals with depression $\geq 25$ years of age, with relatively low ratings of suicidal ideation at baseline, is to reduce rather than increases the risk of suicidal ideation. The outcome in young adults was less clear-cut; although there were some indications that SSRIs might exert beneficial effects in the early phase and harmful effects in the late phase of the trials, both SSRIs and placebo resulted in an end-point rating of suicidality equal to that observed in adults given an SSRI and lower than that observed in adults given placebo.
Jakob Näslund $\mathrm{MD}$, PhD, Fredrik Hieronymus, $\mathrm{MD}$, Alexander Lisinski, $\mathrm{MD}$ Department of Pharmacology, Institute of Neuroscience and Physiology, University of Gothenburg, Gothenburg Sweden; Staffan Nilsson, PhD, Institute of Mathematical Sciences, Chalmers University of Technology, Gothenburg, Sweden; Elias Eriksson, MedDr, Department of Pharmacology, Institute of Neuroscience and Physiology, University of Gothenburg, Sweden

Correspondence: Elias Eriksson, Department of Pharmacology, Institute of Neuroscience and Physiology, Sahlgrenska Academy, University of Gothenburg, POB 431, SE 40530 Gothenburg, Sweden. Email: elias.eriksson@neuro.gu.se

First received 23 May 2017, final revision 2 Nov 2017, accepted 8 Nov 2017

\section{Supplementary material}

Supplementary material is available online at https://doi.org/10. 1192/bjp.2017.24.

\section{Funding}

The study was supported by the Swedish Medical Research Council, Bertil Hållsten's Foundation, Söderberg's Foundation, the Sahlgrenska University Hospital and the Swedish Brain Foundation. The funders had no role in study design, analysis, decision to publish or preparation of the manuscript.

\section{Acknowledgements}

We thank H Lundbeck, GSK and Pfizer for kindly providing us with patient data from the included trials.

\section{References}

1 Teicher MH, Glod C, Cole JO. Emergence of intense suicidal preoccupation during fluoxetine treatment. Am J Psychiatry 1990; 147: 207-10.

2 Healy D, Aldred G. Antidepressant drug use \& the risk of suicide. Int Rev Psychiatry 2005; 17: 163-72.

3 Fergusson D, Doucette S, Glass KC, Shapiro S, Healy D, Hebert P, et al. Association between suicide attempts and selective serotonin reuptake inhibitors: systematic review of randomised controlled trials. BMJ 2005; 330: 396.

4 Gunnell D, Saperia J, Ashby D. Selective serotonin reuptake inhibitors (SSRIS) and suicide in adults: meta-analysis of drug company data from placebo controlled, randomised controlled trials submitted to the MHRA's safety review. BMJ 2005; 330: 385

5 Valuck RJ, Libby AM, Anderson HD, Allen RR, Strombom I, Marangell LB, et al. Comparison of antidepressant classes and the risk and time course of suicide attempts in adults: propensity matched, retrospective cohort study. $\mathrm{Br} \mathrm{J}$ Psychiatry 2016; 208: 271-9.

6 Cheung K, Aarts N, Noordam R, van Blijderveen JC, Sturkenboom MC, Ruiter R et al. Antidepressant use and the risk of suicide: a population-based cohort study. J Affect Disord 2015; 174: 479-84.

7 Garlow SJ, Kinkead B, Thase ME, Judd LL, Rush AJ, Yonkers KA, et al. Fluoxetine increases suicide ideation less than placebo during treatment of adults with minor depressive disorder. J Psychiatr Res 2013; 47: 1199-203.

8 Hammad TA, Laughren TP, Racoosin JA. Suicide rates in short-term randomized controlled trials of newer antidepressants. J Clin Psychopharmacol 2006; 26: 203-7.

9 Jakobsen JC, Katakam KK, Schou A, Hellmuth SG, Stallknecht SE, Leth-Møller K, et al. Selective serotonin reuptake inhibitors versus placebo in patients with major depressive disorder. A systematic review with meta-analysis and trial sequential analysis. BMC Psychiatry 2017; 17: 58.

10 Leon AC, Keller MB, Warshaw MG, Mueller TI, Solomon DA, Coryell W, et al. Prospective study of fluoxetine treatment and suicidal behavior in affectively ill subjects. Am J Psychiatry. 1999; 156: 195-201.

11 Khan A, Khan S, Kolts R, Brown WA. Suicide rates in clinical trials of SSRIs, other antidepressants, and placebo: analysis of FDA reports. Am J Psychiatry 2003; 160: 790-2.

12 Beasley Jr CM, Ball SG, Nilsson ME, Polzer J, Tauscher-Wisniewski S, Plewes J, et al. Fluoxetine and adult suicidality revisited: an updated meta-analysis using expanded data sources from placebo-controlled trials. J Clin Psychopharmacol 2007; 27: 682-6.

13 Gibbons RD, Brown CH, Hur K, Davis JM, Mann JJ. Suicidal thoughts and behavior with antidepressant treatment: reanalysis of the randomized 
placebo-controlled studies of fluoxetine and venlafaxine. Arch Gen Psychiatry 2012; 69: 580-7.

14 Gibbons RD, Brown $\mathrm{CH}$, Hur K, Marcus SM, Bhaumik DK, Mann JJ. Relationship between antidepressants and suicide attempts: an analysis of the Veterans Health Administration data sets. Am J Psychiatry 2007; 164: 1044-49.

15 Grunebaum MF, Ellis SP, Li S, Oquendo MA, Mann JJ. Antidepressants and suicide risk in the United States, 1985-1999. J Clin Psychiatry 2004; 65: 1456-62.

16 Isacsson G. Suicide trends and antidepressants. Br J Psychiatry 2007; 190: 79.

17 Milane MS, Suchard MA, Wong ML, Licinio J. Modeling of the temporal patterns of fluoxetine prescriptions and suicide rates in the United States. PLOS Med 2006; 3: e190.

18 Khan A, Schwartz K. Suicide risk and symptom reduction in patients assigned to placebo in duloxetine and escitalopram clinical trials: analysis of the FDA summary basis of approval reports. Ann Clin Psychiatry 2007; 19: 31-6.

19 Barbui C, Esposito E, Cipriani A. Selective serotonin reuptake inhibitors and risk of suicide: a systematic review of observational studies. CMAJ 2009; 180 291-7.

20 Stone $\mathrm{M}$, Laughren $\mathrm{T}$, Jones $\mathrm{ML}$, Levenson $\mathrm{M}$, Holland $\mathrm{PC}$, Hughes $\mathrm{A}$, et al Risk of suicidality in clinical trials of antidepressants in adults: analysis of proprietary data submitted to US Food and Drug Administration. BMJ 2009; 339: b2880.

21 Martinez C, Rietbrock S, Wise L, Ashby D, Chick J, Moseley J, et al. Antidepressant treatment and the risk of fatal and non-fatal self harm in first episode depression: nested case-control study. BMJ 2005; 330: 389.

22 Hammad TA, Laughren T, Racoosin J. Suicidality in pediatric patients treated with antidepressant drugs. Arch Gen Psychiatry 2006; 63: 332-9.

23 Rabkin JG, Markowitz JS, Stewart J, McGrath P, Harrison W, Quitkin FM, et al. How blind is blind? Assessment of patient and doctor medication guesses in placebo-controlled trial of imipramine and phenelzine. Psychiatry Res 1986; 19 : $75-86$

24 Petkova E, Quitkin FM, McGrath PJ, Stewart JW, Klein DF. A method to quantify rater bias in antidepressant trials. Neuropsychopharmacol 2000; 22 $559-65$.

25 Margraf J, Ehlers A, Roth WT, Clark DB, Sheikh J, Agras WS, et al. How "blind" are double-blind studies? J Consult Clin Psychol 1991; 59: 184-7.
26 Thomson R. Side effects and placebo amplification. Br J Psychiatry 1982; 140: 64-8.

27 Perlis RH, Beasley Jr CM, Wines Jr JD, Tamura RN, Cusin C, Shear D, et al. Treatment-associated suicidal ideation and adverse effects in an open, multicenter trial of fluoxetine for major depressive episodes. Psychother Psychosom 2007; 76: 40-6.

28 Szanto K, Mulsant BH, Houck PR, Dew MA, Dombrovski A, Pollock BG, et al. Emergence, persistence, and resolution of suicidal ideation during treatment of depression in old age. J Affect Disord 2007; 98: 153-61.

29 Seemüller F, Riedel M, Obermeier M, Bauer M, Adli M, Mundt C, et al. The controversial link between antidepressants and suicidality risks in adults: data from a naturalistic study on a large sample of in-patients with a major depressive episode. Int J Neuropsychopharmacol 2009; 12: 181-9.

30 Beasley Jr CM, Dornseif BE, Bosomworth JC, Sayler ME, Rampey Jr AH, Heiligenstein $\mathrm{JH}$, et al. Fluoxetine and suicide: a meta-analysis of controlled trials of treatment for depression. BMJ 1991; 303: 685-92.

31 Nakagawa A, Grunebaum MF, Ellis SP, Oquendo MA, Kashima H, Gibbons RD, et al. Association of suicide and antidepressant prescription rates in Japan, 1999-2003. J Clin Psychiatry 2007; 68: 908-16.

32 Friedman RA. Antidepressants' black-box warning-10 years later. N Eng J Med 2014; 371: 1666-8.

33 Hieronymus F, Nilsson S, Eriksson E. A mega-analysis of fixed-dose trials reveals dose-dependency and a rapid onset of action for the antidepressant effect of three selective serotonin reuptake inhibitors. Transl Psychiatry 2016; 6: e834-7.

34 Näslund J, Hieronymus F, Emilsson JF, Lisinski A, Nilsson S, Eriksson E. Incidence of early anxiety aggravation in trials of selective serotonin reuptake inhibitors in depression. Acta Psychiatr Scand 2017; 136: 343-351.

35 Green KL, Brown GK, Jager-Hyman S, Cha J, Steer RA, Beck AT. The predictive validity of the beck depression inventory suicide item. J Clin Psychiatry 2015; 76: $1683-86$

36 Desseilles M, Perroud N, Guillaume S, Jaussent I, Genty C, Malafosse A, et al. Is it valid to measure suicidal ideation by depression rating scales? J Affect Disord 2012; 136: 398-404. 